\title{
Free-free opacity in dense plasmas with an average atom model
}

\author{
N. R. Shaffer, ${ }^{1}$ N. G. Ferris, ${ }^{2}$ J. Colgan,${ }^{3}$ D. P. Kilcrease,${ }^{3}$ and C. E. Starrett $^{3, *}$ \\ ${ }^{1}$ Department of Physics and Astronomy, University of Iowa, Iowa City, IA 52242 \\ ${ }^{2}$ Dickinson College, Carlisle, PA 17013 \\ ${ }^{3}$ Los Alamos National Laboratory, P.O. Box 1663, Los Alamos, NM 87545, U.S.A.
}

(Dated: February 21, 2017)

\begin{abstract}
A model for the free-free opacity of dense plasmas is presented. The model uses a previously developed average atom model, together with the Kubo-Greenwood model for optical conductivity. This, in turn, is used to calculate the opacity with the Kramers-Kronig dispersion relations. Comparisons to other methods for dense deuterium results in excellent agreement with DFT-MD simulations, and reasonable agreement with a simple Yukawa screening model corrected to satisfy the conductivity sum rule. Comparisons against the very recent experiments of Kettle et al for dense aluminum also reveal very good agreement, in contrast to existing models. Weaknesses in the model are also highlighted.
\end{abstract}

Keywords: opacity, warm dense matter, average atom model

\section{INTRODUCTION}

In the regime of dense plasmas, the influence of the plasma environment on the opacity becomes significant. Recently there has been a particular focus on the freefree contribution to opacity [1-5]. This is driven in part by its relevance for Inertial Confinement Fusion (ICF) modeling [1] but also due its astrophysical relevance in the solar core [6] and the accuracy of astrochonology with white dwarf stars [7,8], as well as the possibility of its direct measurement at free electron laser facilities such as the FLASH XUV-FEL at Hamburg [5]. Very recently, measurements of the free-free contribution to opacity for solid density aluminum at $1 \mathrm{eV}$ and room temperature have been published [4]. These latest experiments were found to disagree with recent efforts to improve modeling of this feature $[3,5]$.

The free-free component of opacity has traditionally been modeled using Kramers' classical cross section [9] modified by a so-called Gaunt factor to account for quantum effects [10]. The quality of the approximation for the Gaunt factor then determines the accuracy of the free-free opacity. The Gaunt factor should, in principle, be calculated directly from free (or continuum) wavefunctions in the presence of the nucleus and bound electrons, with density and temperature effects incorporated through the ionic structure factor and partial ionization fraction of the plasma. Relatively crude approximations for each of these dense plasma effects are often used $[2,3,11,12]$. For example, the ionic structure factor $S(k)$ is taken to be its ideal gas value $(S(k)=1)$ [3], or the scattering potential can be purely Yukawa with bound states taken into account through an average ionization $[2]$.

In this paper we calculate the free-free opacity of dense plasmas with an average atom model $[13,14]$. The

*Electronic address: starrett@lanl.gov average atom model incorporates dense plasma effects self-consistently. It takes into account a realistic ionic structure factor, treats bound and free electrons equally, predicts average ionization and has no ad hoc continuum lowering model. The average atom model is first used to calculate the electronic structure of an atom in the plasma, and then the optical conductivity is calculated using the Kubo-Greenwood formalism [15-18]. The Kramers-Kronig dispersion relations are then used to calculate an index of refraction and hence the opacity.

Calculations based on this model are compared to Density Functional Theory Molecular Dynamics (DFT-MD) simulations for warm dense deuterium at conditions relevant to ICF experiments [1]. We also compare to a recent model [2] that assumes Yukawa screening, sets $S(k)=1$, and determines the screening length based on an average ionization model [19]. This model is relevant because it is used in the Los Alamos ATOMIC code [20, 21] and recent opacity tables. ATOMIC is a multi-purpose opacity and kinetics code that has been recently used to generate new Los Alamos OPLIB opacity tables for hydrogen through zinc [22]. ATOMIC uses atomic data from the Los Alamos suite of atomic physics codes [23] and an equation-of-state (known as ChemEOS) based on a chemical picture [19]. The generated opacity tables are required to cover very large ranges of temperature and density and thus need to use atomic structure and EOS models that are robust and reasonably well-behaved over a number of regions in which different pieces of physics are important.

We also use the present average atom model to calculate the opacity of dense aluminum. We compare to experimental measurements of the opacity of solid density aluminum that are sensitive to the free-free opacity. Under the conditions considered, aluminum is not fully ionized and has significant bound structure. The average atom model used is based on DFT and suffers from the well known band gap problem of DFT. This means that the bound state energies are known to be incorrect. This could presumably be fixed in the usual way with the 
trick $[24,25]$ of replacing the bound-bound DFT interaction energy with the corresponding Hartree-Fock energy. However, we do not attempt this here. Another problem with the present treatment of bound-bound and boundfree transitions is that we do not account for different configurations, i.e. we use only the average atom electronic structure and do not calculate the energy of the different ion configurations. This compounds the band-gap problem. Moreover, it means that at very low density, where many ion stages make important and well separated contributions to the opacity, the model will show just one contribution (one line). However at higher densities the issue becomes less important as the opacity is dominated by one ion stage (see, for example, reference [24]). The last major weakness of the present models approach to transitions involving bounds states is a relatively crude treatment of line broadening. We use a Lorentz profile for bound-bound transitions with a width given by the method prescribed in [18]. Since our main aim is to model the free-free opacity we tolerate these weaknesses at present.

Others have used average atom models to calculate opacity in the dense plasma regime (for example $[6,24,26-28])$. These methods have variously accounted for different configurations, and corrected the DFT energies using the usual trick [25]. However, the free-free opacity has not been their focus and they did not address the issues relating to its calculations, viz. the ionic structure, satisfaction of sum rules, and the inclusion of a the index of refraction.

The structure of this paper is as follows. In section II we describe the average atom model and how to calculate opacity with it. In section III we give the numerical results for opacity of warm dense deuterium and compare to the DFT-MD and ATOMIC results. We find a straightforward way to improve the ATOMIC results and this is described. We also show results for aluminum. First for cold aluminum where we are able to compare to experiments on the opacity and the index of refraction. Then we show a series of opacities for aluminum at $5 \mathrm{eV}$ from $1 / 100^{t h}$ to 1 times solid density and compare to the ATOMIC code. Finally in section IV we draw our conclusions. Unless otherwise stated, Hartree atomic units are used throughout in which $\hbar=m_{e}=e=1$ )

\section{DESCRIPTION OF THE AVERAGE ATOM APPROACH TO OPACITIES}

\section{A. Description of the Average Atom model}

The average atom model that we are using has been described in extensive detail elsewhere $[13,14]$. Here we give a only brief summary. The model finds the electron density and effective potential felt by an electron selfconsistently. The Schrödinger equation is solved using the effective potential to give bound and free eigenvalues and wavefunctions. The states are occupied according to the Fermi-Dirac occupation factor $f(\epsilon, \mu)$, where $\epsilon$ is the energy of the state and $\mu$ the electronic chemical potential.

We solve two self-consistent field problems. In the first (the "full" model) the effective potential $V_{N e}^{e f f}(r)$ is given by

$$
\begin{aligned}
V_{N e}^{e f f}(r)= & -\frac{Z}{r}+\int d^{3} r^{\prime} \frac{\left(n_{e}^{f u l l}\left(r^{\prime}\right)-n_{e}^{0} g_{I I}\left(r^{\prime}\right)\right)}{\left|\boldsymbol{r}-\boldsymbol{r}^{\prime}\right|} \\
& +V_{e e}^{x c}\left[n_{e}^{f u l l}(r)\right]-V_{e e}^{x c}\left[n_{e}^{0}\right]
\end{aligned}
$$

where $Z$ is the nuclear charge, $n_{e}^{f u l l}(r)$ is the electron density, $n_{e}^{0}$ is the free electron density, $g_{I I}(r)$ is the ionion pair distribution function, and $V_{e e}^{x c}(r)$ is the electron exchange potential

$$
V_{e e}^{x c}[n]=-\left(\frac{3 n}{\pi}\right)^{\frac{1}{3}} .
$$

The electron density is calculated from the wavefunctions which separate naturally into a negative energy, discrete, bound state spectrum and a continuum of positive energy states. The electron density is thus given by

$$
n_{e}^{f u l l}(r)=n_{e}^{b}(r)+n_{e}^{c}(r)
$$

where

$$
n_{e}^{b}(r)=\sum_{n, l \in B} f\left(\epsilon_{n, l}, \mu\right) \frac{2(2 l+1)}{4 \pi}\left|\frac{y_{\epsilon_{n, l}}(r)}{r}\right|^{2}
$$

and

$$
n_{e}^{c}(r)=\int_{0}^{\infty} d \epsilon f(\epsilon, \mu) \sum_{l=0}^{\infty} \frac{2(2 l+1)}{4 \pi}\left|\frac{y_{\epsilon, l}(r)}{r}\right|^{2}
$$

where the $y$ 's are the radial components of the wavefunctions [13]. The chemical potential is determined by requiring the ion-sphere to be charge neutral, i.e. the chemical potential is adjusted until

$$
\int_{V} n_{e}(r) d^{3} r=Z
$$

is satisfied, where $V$ is the volume of the ion sphere. By solving equations (1) to (6) iteratively until selfconsistent, we get an electron density $n_{e}^{\text {full }}(r)$, a chemical potential $\mu$, bound and free wavefunctions and an effective scattering potential $V_{N e}^{e f f}(r)$.

The second self-consistent field problem (the "external" model) attempts to calculate the part of $n_{e}^{\text {full }}$ that is due only to the external system (i.e. the $g_{I I}(r)$ ). The ultimate aim is to isolate an electron density due to the central nucleus only; this is known as the pseudoatom density $n_{e}^{P A}(r)$. To calculate the part of $n_{e}^{\text {full }}(r)$ due only to $g_{I I}(r)$ we self-consistently solve the same set of equations (1) to (3) but without the nucleus at the origin (i.e. set $Z=0$ ), and keeping $\mu$ fixed. The resulting 
electron density $n_{e}^{e x t}(r)$ is due only to $g_{I I}(r)$, and hence we define the pseudoatom density as

$$
n_{e}^{P A}(r)=n_{e}^{f u l l}(r)-n_{e}^{e x t}(r)
$$

The pseudoatom density is the electron density due to only one nucleus in the plasma. The total electron density of the plasma would then be a superposition of pseudoatom densities at each nuclear site [29]. The reason we wish to have it in the present model is that it can be coupled to the so called quantum-Ornstein-Zernike equations to give the ion-ion pair distribution function $g_{I I}(r)$. We do not give the details here, see reference [14]. This $g_{I I}(r)$ can be fed back into equation (1) and the whole process repeated until converged. However, it was shown in reference [14] that only one iteration is sufficient, i.e. we just need to solve the two self-consistent field problems once using an initial guess for $g_{I I}(r)=\Theta(r-R)$. Here $\Theta$ is the Heaviside step function at the ion sphere radius. As shown in reference [14], the $g(r)$ from this one step procedure is almost identical to the full self-consistent procedure.

This average atom model thus results in a consistent set of physical properties, including bound and free states and their occupations, scattering potential, average ionization, chemical potential and ionic distribution function.

\section{B. Calculation of opacity from average atom model}

The opacity is equal to the absorption coefficient plus an electron scattering term. This latter term is negligible for the results presented here and we will use the terms absorption and opacity interchangeably. The absorption coefficient $\alpha(\omega)$ at photon energy $\hbar \omega$ is related to the real part of the optical conductivity $\sigma_{1}(\omega)$ and the real part of the index of refraction $n(\omega)$ by

$$
\alpha(\omega)=\frac{4 \pi \sigma_{1}(\omega)}{n(\omega) c}
$$

where $c$ is the speed of light. $n(\omega)$ is related to the real and imaginary parts of the dielectric function $\epsilon_{1}(\omega)$ and $\epsilon_{2}(\omega)$ respectively [18], via

$$
n(\omega)=\sqrt{\left(\frac{\epsilon_{1}(\omega)+|\epsilon(\omega)|}{2}\right)}
$$

where $\epsilon=\epsilon_{1}+\imath \epsilon_{2}$. These are in turn related to the real and imaginary parts of the optical conductivity $\left(\sigma_{1}\right.$ and $\left.\sigma_{2}\right)$

$$
\begin{aligned}
\epsilon_{1}(\omega) & =1-\frac{4 \pi \sigma_{2}(\omega)}{\omega} \\
\epsilon_{2}(\omega) & =\frac{4 \pi \sigma_{1}(\omega)}{\omega}
\end{aligned}
$$

Finally, $\sigma_{2}$ is related to $\sigma_{1}$ by a Kramers-Kronig relation

$$
\sigma_{2}(\omega)=-\frac{2 \omega}{\pi} P \int_{0}^{\infty} \frac{\sigma_{1}(\omega)}{\omega^{2}-x^{2}} d x
$$

where $P$ denotes the Cauchy principal value integral. Thus, with knowledge of only the real part of the optical conductivity $\sigma_{1}(\omega)$ we can calculate the absorption coefficient $\alpha(\omega)$. This procedure, i.e. getting $\alpha(\omega)$ from $\sigma_{1}(\omega)$, is qualitatively the same as used in DFT-MD simulations $[1,7]$.

The method of calculating $\sigma_{1}(\omega)$ is described in reference [15]. In brief, we evaluate

$$
\begin{aligned}
\sigma_{1}(\omega)= & -2 \pi n_{I}^{0} \int d \epsilon \frac{f\left(\epsilon_{n}, \mu\right)-f\left(\epsilon_{m}, \mu\right)}{\omega} \int d \hat{\boldsymbol{k}}_{m} \int d \hat{\boldsymbol{k}}_{n} \\
& \times\left|J_{m n}\right|^{2} S\left(\left|\boldsymbol{k}_{n}-\boldsymbol{k}_{m}\right|\right)
\end{aligned}
$$

where $S(k)$ is the ion-ion structure factor, $\epsilon_{m}=\epsilon+\omega$, $\epsilon_{n}=\epsilon$, and $n_{I}^{0}=1 / V$. $J_{m n}$ is the matrix element between the initial and final state

$$
J_{m n} \equiv \int d^{3} r \psi_{\boldsymbol{k}_{m}}^{*}(\boldsymbol{r}) \hat{\boldsymbol{v}}_{z} \psi_{\boldsymbol{k}_{n}}(\boldsymbol{r})
$$

in the velocity gauge. With the wave functions $\psi$, the chemical potential $\mu$, and structure factor $S(k)$ from the average atom model $\left(S(k)\right.$ is simply related to $\left.g_{I I}(r)\right)$, $\sigma_{1}(\omega)$ can thus be calculated.

As written $\sigma_{1}(\omega)$ diverges as $\omega^{-2}$ when $\omega \rightarrow 0$. This well understood problem is rectified in an ad hoc way by multiplying the integrand in the formula (13) by a Drude scaling factor $\omega^{2} /\left(\nu^{2}+\omega^{2}\right)$. This regularizes $\sigma_{1}$ so that it gives a finite (and reasonable) value at $\omega=0$. The parameter $\nu$ is the energy independent collision frequency and is determined by requiring that the free-free contribution to the optical conductivity $\sigma_{1}^{f f}(\omega)$ satisfies the conductivity sum rule

$$
\int d \omega \sigma_{1}^{f f}(\omega)=\frac{\pi}{2} \frac{\bar{Z}}{V}
$$

where $\bar{Z}$ is the average ionization (see reference [14]).

This all seems straightforward, however, there is one complication. As discussed in reference [15] one has a choice of scattering potential in which to calculate the wavefunctions $\psi$. Reasonable arguments can be made for either using the average atom potential $V_{N e}^{e f f}(r)$ or the so-called pseudoatom potential

$$
\begin{aligned}
V_{P A}(r)= & -\frac{Z}{r}+\int d \boldsymbol{r}^{\prime} \frac{n_{e}^{P A}\left(r^{\prime}\right)}{\left|\boldsymbol{r}-\boldsymbol{r}^{\prime}\right|} \\
& +V_{e e}^{x c}\left[n_{e}^{f u l l}(r)\right]-V_{e e}^{x c}\left[n_{e}^{e x t}(r)\right] .
\end{aligned}
$$

Empirically we find that $V^{P A}(r)$ gives better results for the dc conductivity $\left(\sigma_{1}(0)\right)$ compared to ab initio simulations at high temperatures and densities, whereas $V_{N e}$ gives better results as we approach normal (solid) densities and cold temperatures [15, 30]. Without further investigation we will use the two potentials where experience suggests they should be reasonable. Clearly, for the average atom method to be used more generally this issue will have to be resolved. Where we have used the average atom potential the results are labeled AA-KG, and where we have use the pseudoatom potential the results are labeled PA-KG. 


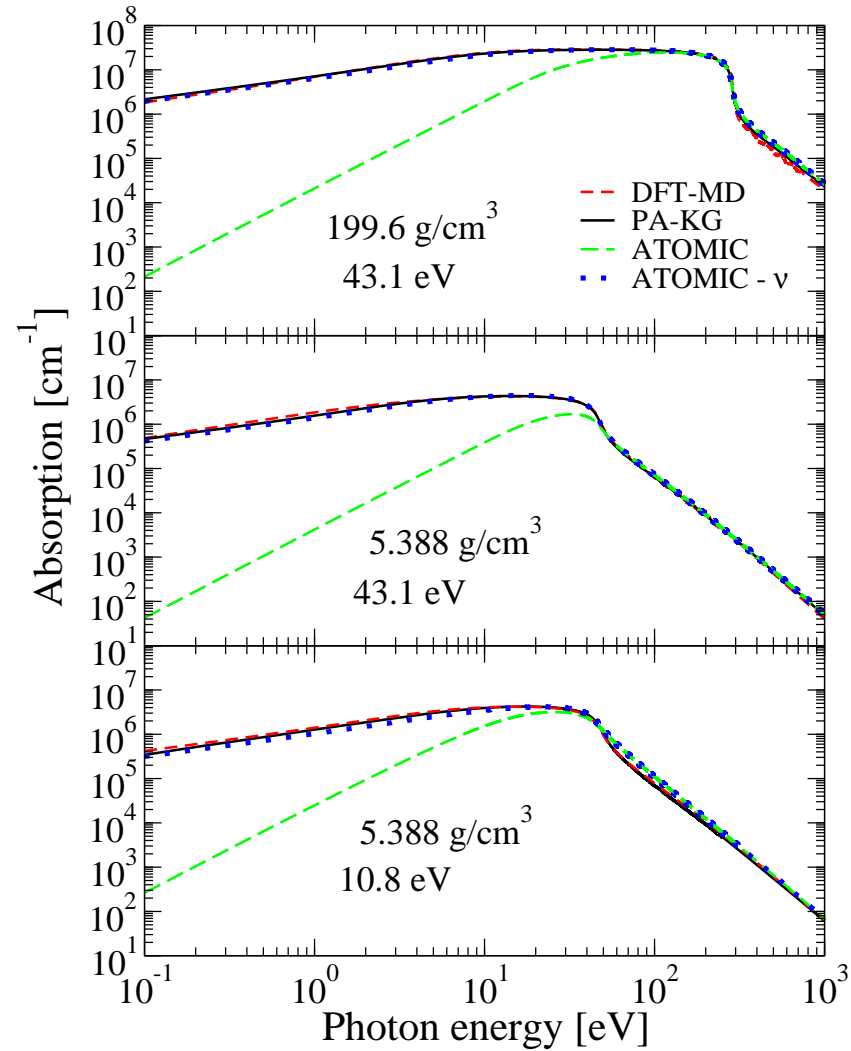

FIG. 1: (Color online) The absorption coefficient of deuterium plasmas. DFT-MD is from reference [1], ATOMIC uses the model given in reference [2], while ATOMIC- $\nu$ and PA-KG are described in the text. The essential difference between ATOMIC and ATOMIC- $\nu$ is that the latter is required to satisfy the conductivity sum rule.

\section{RESULTS}

\section{A. Warm dense deuterium}

In figure 1 we show the opacity of warm dense deuterium using the pseudoatom potential (PA-KG). We compare to DFT-MD simulation results of reference [1]. The DFT-MD method is also a DFT based method, with the opacity calculated from the real part of the optical conductivity as for the average atom method used here. The main physical difference is that the DFT-MD simulations solve for the wavefunctions in a many atom system, subject to periodic boundary conditions, whereas the average atom method has just one atom in jellium. DFTMD therefore should be more physically realistic than the average atom model. However, the method has a number of important practical limitations due to its extreme computational expense. The two main limitations are that it is too computationally expensive to apply when the temperature becomes comparable to, or higher than the Fermi temperature, and that a pseudopotential is generally used to represent any core electrons that are present.

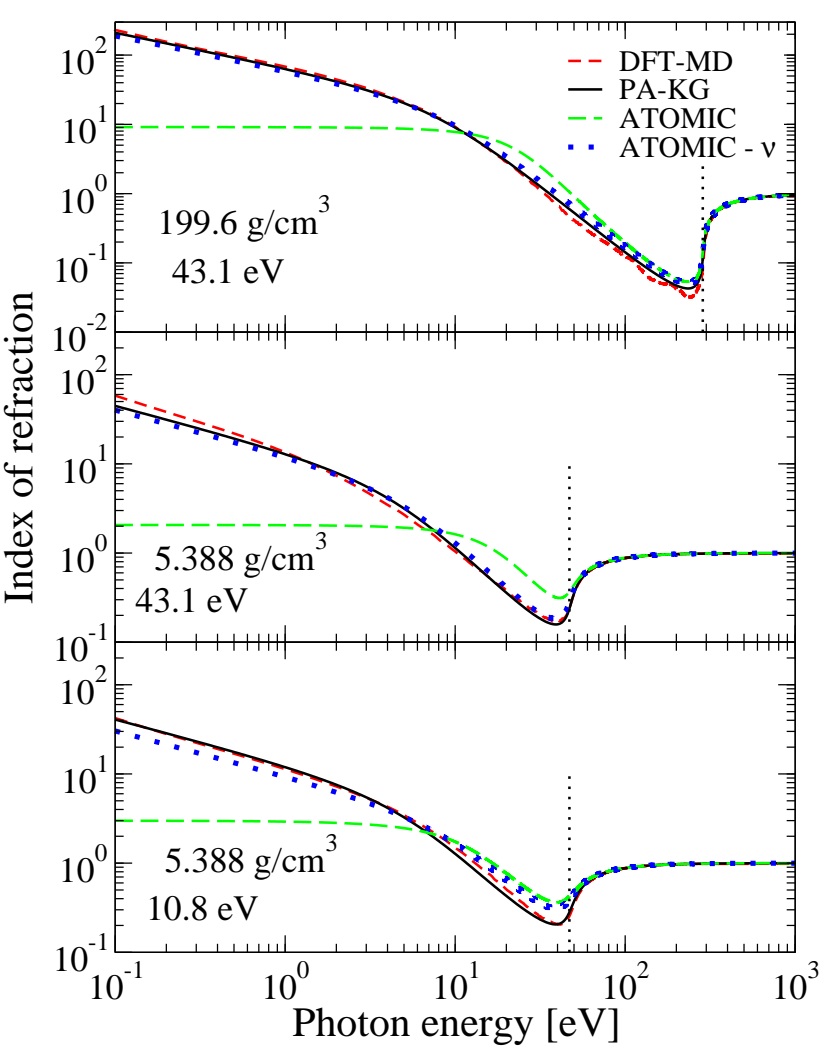

FIG. 2: (Color online) The index of refraction of deuterium plasmas. DFT-MD is from reference [1], ATOMIC uses the model given in reference [2], while ATOMIC- $\nu$ and PA-KG are described in the text. The essential difference between ATOMIC and ATOMIC- $\nu$ is that the latter is required to satisfy the conductivity sum rule. The vertical dotted black lines indicate the plasma frequency for each case.

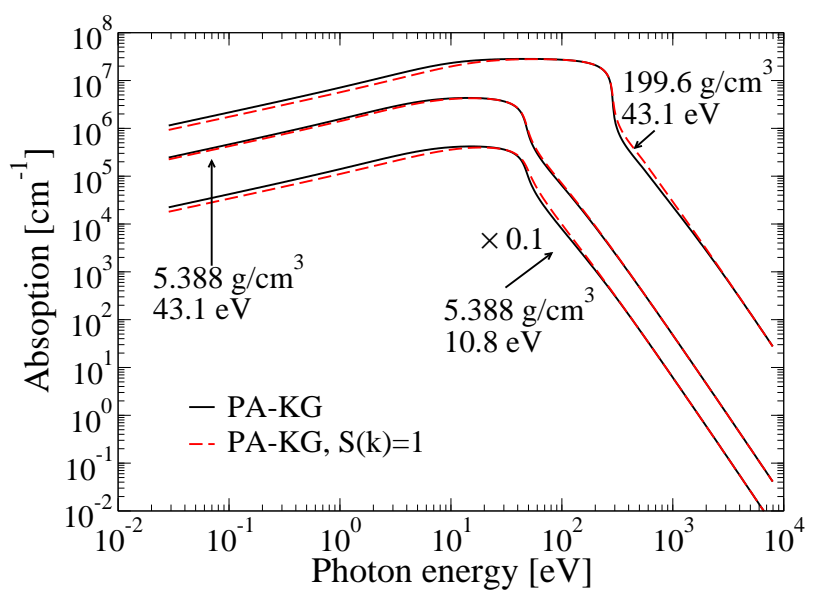

FIG. 3: (Color online) Absorption coefficient from the PAKG model (see text). The solid black line is with a calculated (realistic) ion structure factor $S(k)$, whereas the dashed red line uses $S(k)=1$. The $5.388 \mathrm{~g} / \mathrm{cm}^{3}$ and $10.8 \mathrm{eV}$ curve has been multiplied by a factor of 0.1 for clarity. 


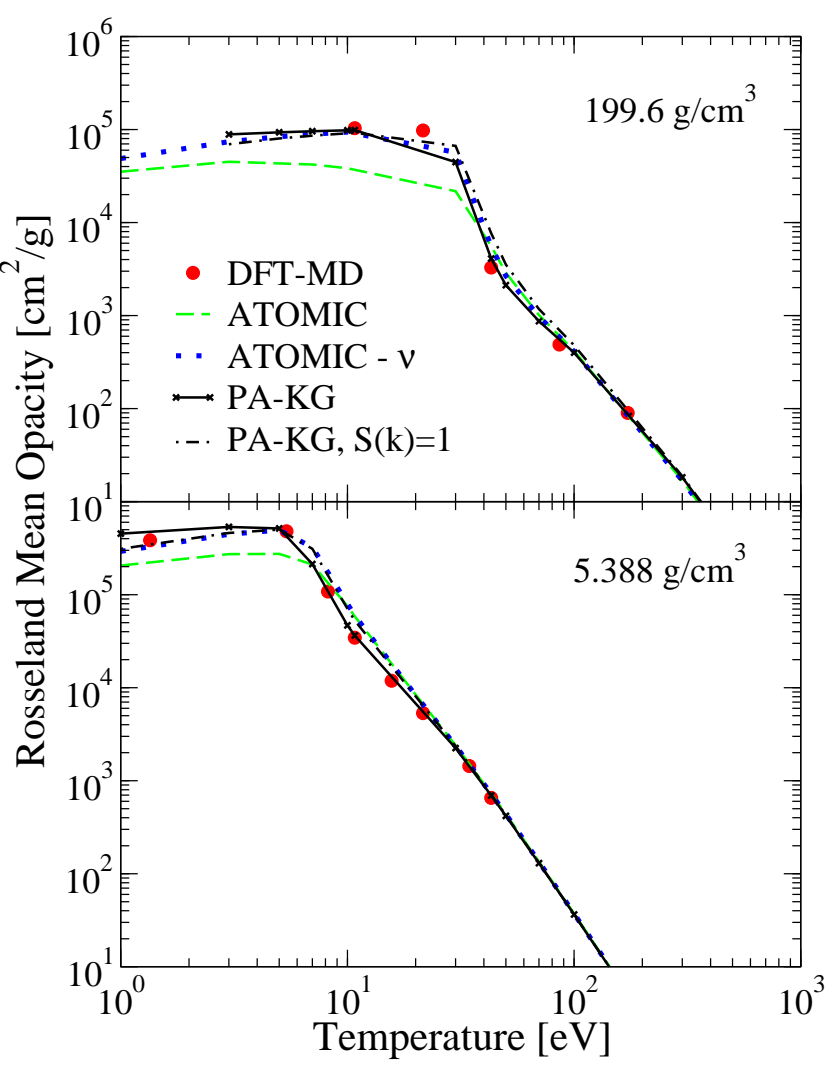

FIG. 4: (Color online) Rosseland mean opacity of deuterium. DFT-MD is from reference [1], ATOMIC uses the model given in reference [2], while ATOMIC- $\nu$ and PA-KG are described in the text. The essential difference between ATOMIC and ATOMIC- $\nu$ is that the latter is required to satisfy the conductivity sum rule.

This latter simplification must be carefully used to avoid artifacts. Nevertheless, for the present comparison (figure 1) DFT-MD should be the most accurate method presented and we can use it as a benchmark.

The PA-KG results agree very well with the DFT-MD calculations, for all three conditions. This is remarkable given the very different approximations that go into the two approaches. In contrast, using the method presented in reference [2] (labeled ATOMIC) the agreement becomes poorer. In particular, at low photon frequencies the ATOMIC result strongly departs from both DFT-MD and PA-KG. To understand this significant difference let us examine the method of reference [2] (see also reference [3]) more closely. The expression of the optical conductivity in this method can be obtained using equation (8) above and equations (3.1) and (3.2) of reference [3],

$$
\begin{aligned}
\sigma_{1}(\omega)= & \frac{c}{4 \pi}\left(1-e^{-\hbar \omega / T}\right) \frac{\omega^{2}}{\omega^{2}+\nu(\omega)^{2}} \\
& \times g(\omega, T) \alpha_{k}(\omega)
\end{aligned}
$$

(see reference [3] for an explanation of the terms in this equation). It is straightforward, if lengthy, to show that this is equal to the expression given in equation (13) above provided that 1$) S(k)$ is set equal to 1,2$)$ the scattering potential (and thus the wavefunctions) are the same, 3) the chemical potential and the corresponding average electron density are the same, and 4) the photon frequency dependent collision frequency in equation (17) is replaced with an energy independent collision frequency chosen so that $\sigma_{1}(\omega)$ satisfies the conductivity sum rule equation (15).

Implementing this last change (point 4) in the model of Armstrong et al [2], results in the curve in figure 1 labeled ATOMIC- $\nu$. Clearly, the agreement is substantially improved. For clarity we repeat, this new result is the same model as given in reference [2] except that the collision frequency has been made energy independent and chosen to satisfy the sum rule above (equation (15)). It may seem that we have worsened the physical model, after all the collision frequency should be energy dependent. However, it seems that it is more important to ensure that the conductivity sum rule is enforced. One could imagine that an energy dependent collision frequency that has the correct asymptotic behavior as $\omega \rightarrow 0$ and satisfies the sum would be the "best" solution. Steps towards this goal have been taken [31, 32] but issues remain. The simple step we have taken to enforce the sum rule in the ATOMIC model dramatically improves the agreement with the other models and seems to be adequate for present purposes.

In figure 2 we show the index of refraction for the same deuterium cases. Again we see excellent agreement between the PA-KG and DFT-MD methods. The ATOMIC model [2] does not agree well with these two methods below the plasma frequency (as in figure 1). The ATOMIC model with the sum rule enforced (ATOMIC$\nu$ ) shows vastly improved agreement but (much smaller) differences remain. There are two principal sources for this remaining disagreement: 1) the ATOMIC- $\nu$ model uses a Yukawa model with a calculated screening length whereas the average atom model uses a self-consistent potential, and 2) the index of refraction in ATOMIC- $\nu$ uses a Drude model for the index of refraction [3] (with a now energy independent collision frequency) whereas the average atom model calculates the "exact" index of refraction for the given optical conductivity via the KramersKronig relations (equations (9) to (12)).

Figure 3 examines at the influence of the structure factor $S(k)$. We compare calculations that use the calculated $S(k)$ versus those that set $S(k)=1$, both within the context of the average atom model. For the cases in the figure, we see that there is a modest but noticeable effect. The effect is more important for the more strongly coupled cases (i.e. higher density, or lower temperature). This is of course expected, since for weakly coupled cases $S(k) \rightarrow 1$ for all $k$.

Lastly for deuterium, we show in figure 4 the Rosseland 


\begin{tabular}{ccc}
\hline \hline density $\left[\mathrm{g} / \mathrm{cm}^{3}\right]$ & ATOMIC- $\nu$ & AA-KG \\
0.027 & 1.021 & 1.027 \\
0.27 & 1.350 & 1.804 \\
2.7 & 7.285 & 1.771 \\
\hline \hline
\end{tabular}

TABLE I: Aluminum Rosseland mean opacities in $10^{4} \mathrm{~cm}^{2} / \mathrm{g}$ corresponding to the cases in figure 7 . Differences at higher densities are principally due to the treatment of free-free transitions.

mean opacity $\kappa_{R}$ as defined by

$$
\frac{1}{\kappa_{R}}=\frac{\int_{0}^{\infty} d \omega \frac{\partial B(\omega, T)}{\partial T} n(\omega)^{2} \frac{1}{\alpha_{m}(\omega)}}{\int_{0}^{\infty} d \omega \frac{\partial B(\omega, T)}{\partial T} n(\omega)^{2}}
$$

where $\alpha_{m}(\omega)=\alpha(\omega) / \rho$ is the mass absorption coefficient, $\rho$ is the mass density and $B(\omega, T)$ is the Planck distribution function of the photon energies at temperature $T$. We note that definitions of the Rosseland mean vary and that the opacity tables calculated using ATOMIC [22] utilize a different definition [33]. In the figure we see that PA-KG and DFT-MD agree very well for all temperatures and both densities. At high temperatures ATOMIC is also in agreement with these two methods. The temperature derivative of the Planck distribution function $\frac{\partial B(\omega, T)}{\partial T}$ is peaked at around $4 T[7]$ so the higher the temperature the more important are contributions from high photon frequency - a region relatively well modeled by the method of [2] (i.e. ATOMIC), and hence the good agreement seen. At lower temperatures however, low photon frequencies become more important we find that the ATOMIC results depart from the DFT-MD and PA-KG results. The ATOMIC- $\nu$ method again demonstrates considerable improvement over ATOMIC though differences with PA-KG and DFT-MD remain. An accurate treatment of the ionic structure factor $S(k)$ in particular seems to be important for recovering the "kink" at 10 $\mathrm{eV}$ for the $5.388 \mathrm{~g} / \mathrm{cm}^{3}$ case (compare PA-KG, $S(k)=1$ to PA-KG lines).

\section{B. Warm dense aluminum}

In figure 5 we show the mass absorption coefficient for solid density aluminum. For cold (i.e. $\ll 1 \mathrm{eV}$ ) aluminum there are some experimental data: Keenan et al [34] and the very recent measurements of Kettle et al [4]. We also show lines from Henke et al [35] and Gullikson et al $[36,37]$ that are compiled from experimental measurements and theoretical calculations. There is also a single experimental point for $1 \mathrm{eV}$ that is very close to the cold measurement by the same authors (Kettle et al [4]). The top panel shows a focused region to highlight the comparisons. The average atom model described above is designed for plasmas and is not well suited to crystal lattices and cold temperatures. Thus we show a series of AA-KG

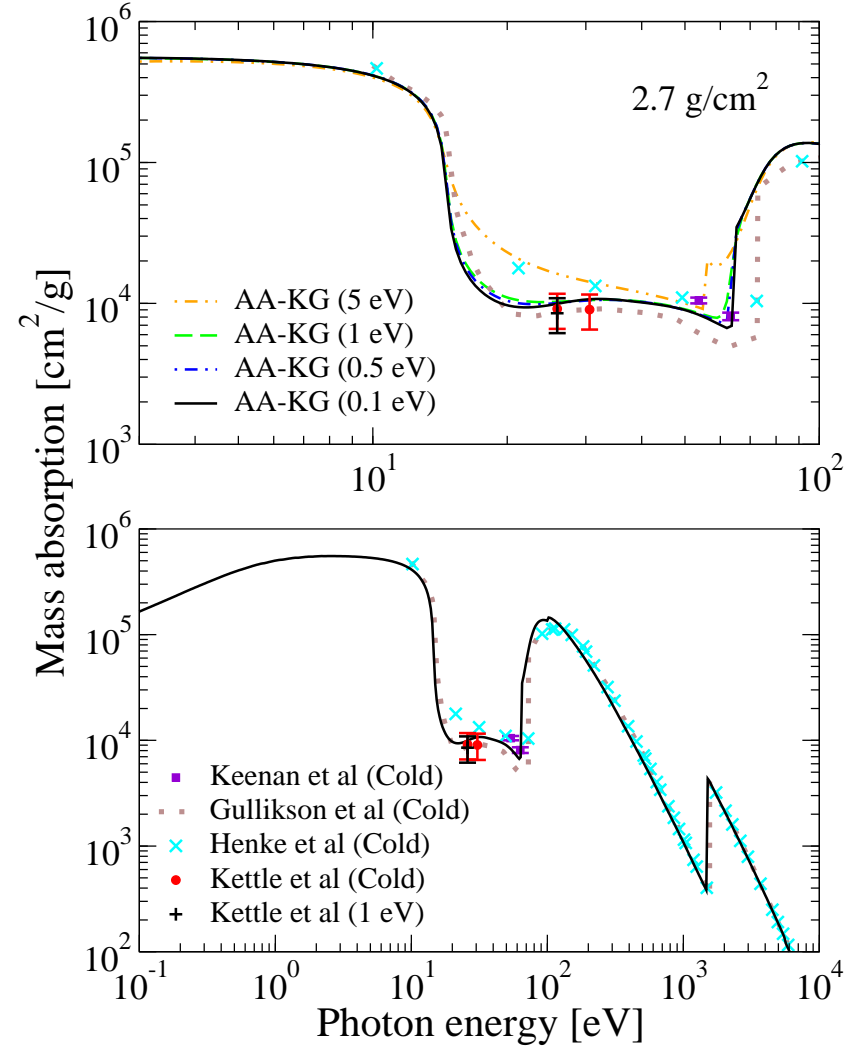

FIG. 5: (Color online) Mass absorption coefficient for aluminum at solid density. The top panel is a focused region of the bottom panel. The experimental data is from references $[4,34-37]$ and is for cold solid aluminum. The single experimental point at elevated temperature $(1 \mathrm{eV})$ is also from reference [4]. The AA-KG model is a plasma model and cold solid conditions are beyond its regime of accuracy. Hence we show a series of AA-KG results for low temperatures to indicate that the result is not very sensitive to temperature below $1 \mathrm{eV}$, thus allowing meaningful comparison to the cold data.

results from $5 \mathrm{eV}$ through to $0.1 \mathrm{eV}$ in temperature. We see very little change in $\alpha_{m}$ below $1 \mathrm{eV}$ in agreement with the experiments of Kettle et al [4]. Hence, even though the average atom method is not shown at the experimental cold conditions, the comparison with the cold data is still meaningful. The agreement of the AA-KG model with the recent Kettle et al measurements is impressive. In contrast, in reference [4] it was shown that the recent models of references [3] and [5] do not agree with both data points (though the model of reference [5] lies within the error pars of the higher temperature point).

The comparison to the Keenan et al data and the lines from Henke et al and Gullikson et al reveals somewhat poorer agreement with AA-KG, though still reasonable given the level of disagreement between these experiments themselves. At the photon energies of the Keenan et al experiment we are quite close to the L shell boundfree edge. As noted earlier the treatment of transitions involving bound states is relatively crude in the present 


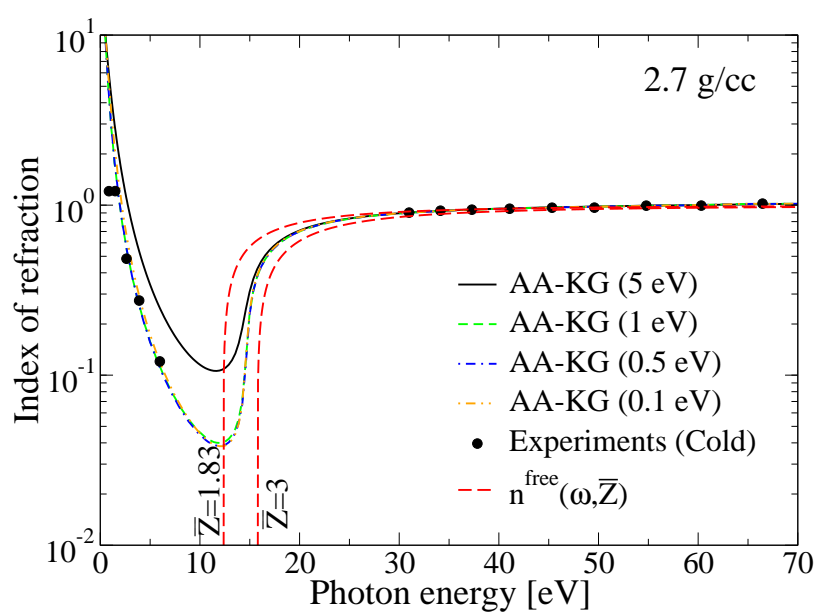

FIG. 6: (Color online) Index of refraction for aluminum at solid density. Experimental data is from reference [36, 38] (see also reference [3]) and is for cold aluminum. We show a series of AA-KG indicating that the result is not very sensitive to temperature below $1 \mathrm{eV}$, thus allowing a meaningful comparison. Also shown is the free electron result $n^{\text {free }}$ that depends on the choice of average ionization $\bar{Z}$.

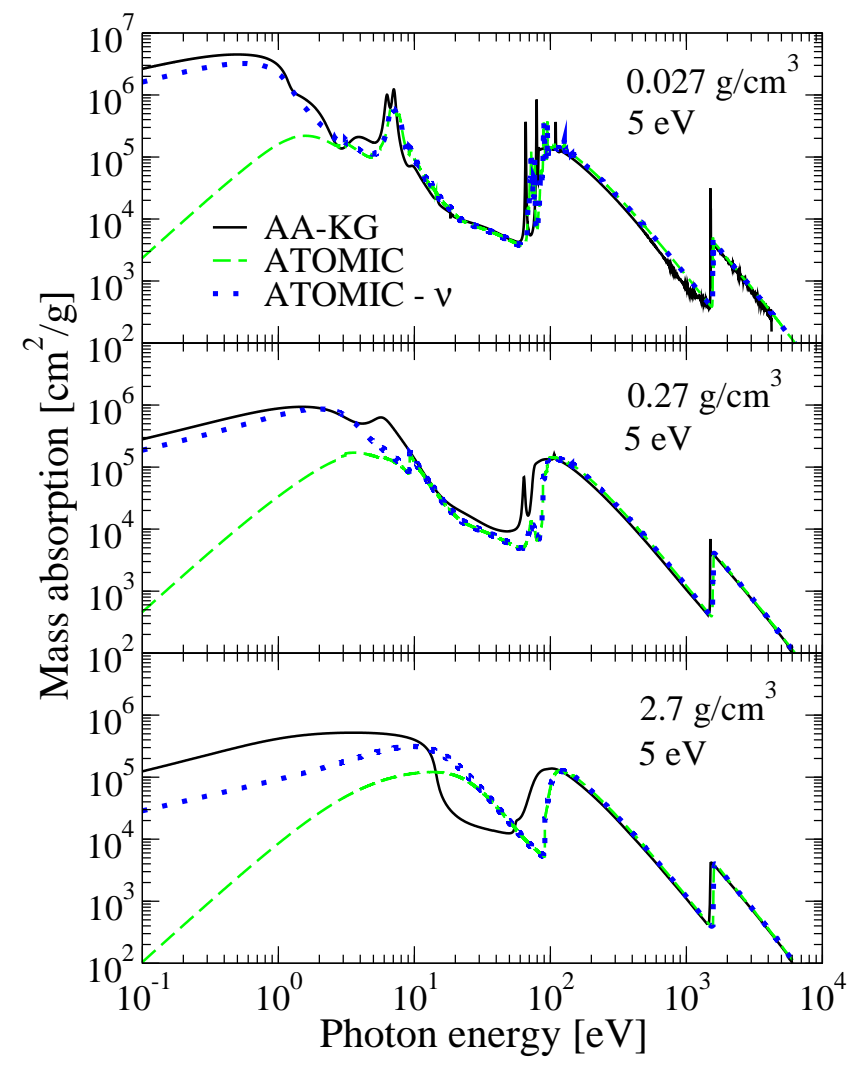

FIG. 7: (Color online) Mass absorption coefficient of aluminum at $5 \mathrm{eV}$ for $1 / 100^{t h}, 1 / 10^{t h}$ and 1 times solid density. Agreement between AA-KG and ATOMIC- $\nu$ is quite good at low density, and becomes poorer at higher densities. Differences are largely due to the treatment of the free-free contribution. model and the position of the edge is almost certainly inaccurate for the reasons mentioned earlier. Overall, these comparisons demonstrate that the AA-KG model is a reasonable method for predicting the opacity of aluminum at solid density.

In figure 6 we show the index of refraction for the same aluminum case as in figure 5 . We compare to experimental measurements [36, 38] (see also reference [3]) for cold solid aluminum. Good agreement between AA-KG and the experimental points is observed both at low photon energy and high. We also show the free electron result for the index of refraction

$$
n^{\text {free }}(\omega)=\sqrt{1-\frac{4 \pi \bar{Z}}{V \omega^{2}}}
$$

which depends on the choice of average ionization $\bar{Z}$. We show $n^{\text {free }}(\omega)$ for two values of $\bar{Z}: \bar{Z}=3$ which corresponds to the total number of continuum electrons per atoms, and $\bar{Z}=1.83$ which corresponds to the number free electrons per atom, i.e. the number of electrons per atom with chemical potential $\mu$ in a zero interaction potential environment (for further discussion see for example $[39,40])$. These two estimates give an upper and lower limit on the actual number of effectively free electrons per atom and the actual result (AA-KG) lies somewhere in between, as expected.

Finally, in figure 7 we show the mass absorption coefficient for aluminum at $5 \mathrm{eV}$ at $1 / 100^{\text {th }}, 1 / 10^{\text {th }}$ and 1 times solid density compared to ATOMIC and ATOMIC- $\nu$. At the lowest density the bound-bound and bound-free features line up well. There is some difference in the energy at which they appear which is likely to be due to the DFT band gap issue in the AA-KG results. ATOMIC and ATOMIC- $\nu$ have a more sophisticated treatment of the bound states at low densities than the average atom model. Clearly ATOMIC- $\nu$ is much closer to AA-KG than ATOMIC, though there are some differences still at the lower photon energies. We note that the model used in ATOMIC- $\nu$ calculates the free states in a Yukawa potential. The influence of bound states is only taken into account by reducing the effective charge of the nucleus, i.e. the free states are not orthogonal to the bound states as they should be (and are in the average atom model). This point was found to be significant in reference [3] in order to find agreement with the cold aluminum experimental data at solid density. This is likely to be the dominant source of difference between AA-KG and ATOMIC- $\nu$ at the low energies. As density is increased the disagreement becomes larger and is again likely to be largely due to the simple treatment of the bound states in the free-free component of the ATOMIC- $\nu$ model. In table I we give the corresponding Rosseland mean opacities. At the lowest density the agreement is good, but as density increases the agreement significantly deteriorates. We note that the temperature derivative of the Plank function peaks near $20 \mathrm{eV}$ for all these cases, hence the free-free differences are a major source of the observed differences in Rosseland mean opacity. 


\section{CONCLUSIONS}

A model for calculating the opacity of dense plasmas with an average atom model has been presented. The model focuses on the free-free component and results for warm dense deuterium are compared to DFT-MD simulation results, with excellent agreement being found. Comparisons to a recent model of Armstrong et al [2] reveal a remarkable discrepancy at low photon frequencies. It was shown that by enforcing a conductivity sum rule in the model of Armstrong et al, a vast improvement with the average atom model and DFT-MD results is found. Calculations for the index of refraction for the same deuterium cases also found good agreement between the average atom model and the DFT-MD results. The model of Armstrong et al (with the sum rule enforced) was also in reasonable agreement. The remaining differences were attributed to the approximate Drude model for the index of refraction used in the Armstrong et al model, whereas the average atom model uses the "exact" Kramers-Kronig relation. We also investigated the effect of a realistic ionic structure factor on the opacity for the deuterium cases. A modest, but noticeable, effect was found in particular for the most strongly coupled cases (i.e. at the highest densities or lower temperatures).

A second set of comparisons, this time for aluminum, was also given. Comparisons for cold, solid density aluminum to experiments that probe free-free opacity revealed that the average atom model was again reasonable. In particular, the average atom model was in agreement with the very recent measurements of Kettle et al [4]. These measurements were at both cold conditions as well as $1 \mathrm{eV}$. The agreement with these measurements was in contrast to the partial disagreement found with comparison to the earlier models of references $[3,5]$. We attribute this to the improved physics model in the average atom treatment, viz. the self consistent treatment of bound and free electrons, and it being a "physical" model as opposed to a "chemical" model. At higher energies than measured by Kettle et al there is also rea- sonable agreement with experiments [34, 35, 37], though not as good. However, the experiments themselves do not agree with each other. Comparisons for the index of refraction for cold, solid aluminum against experimental measurements also reveal excellent agreement, further confirming the accuracy of the average atom model for free-free opacity.

Lastly, a comparison with the ATOMIC code for aluminum at $5 \mathrm{eV}$ at $1 / 100^{\text {th }}, 1 / 10^{\text {th }}$ and 1 times solid density reveals that even with the improved model of Armstrong et al (with the sum rule enforced), agreement on the free-free opacity at the highest density is quite poor. This is due to the use of a simple Yukawa scattering potential in the Armstrong et al model [2] that does not enforce core-valence orthogonality in the wavefunctions (this is enforced in the average atom). Hence, an accurate treatment of free-free opacity in the dense plasma regime requires a consistent treatment of all electrons. These comparisons also reveal the weaknesses in the average atom treatment of transitions involving bound states, i.e. principally the DFT energies of the bound states but also the use of a single average ion stage instead of a proper treatment of distinct ion stages. These known weaknesses could be improved, but our focus in this study is the freefree contribution, so we have not addressed them here.

Once last point of weakness in the average atom model is the choice of scattering potential. We have used two choices where experience suggests that they will give reasonable results. However, a more general approach is desirable and necessary for wide ranging production of free-free opacity tables.

\section{Acknowledgments}

We acknowledge useful conversations with L. Collins, S. Vinko and M. Desjarlais, and thank S. X. Hu for providing the deuterium DFT-MD data. This work was performed under the auspices of the United States Department of Energy under contract DE-AC52-06NA25396 and LDRD number 20150656ECR.
[1] S. X. Hu, L. A. Collins, T. R. Boehly, J. D. Kress, V. N. Goncharov, and S. Skupsky. First-principles thermal conductivity of warm-dense deuterium plasmas for inertial confinement fusion applications. Phys. Rev. E, 89:043105, Apr 2014.

[2] G.S.J. Armstrong, J. Colgan, D.P. Kilcrease, and N.H. Magee Jr. Ab initio calculation of the nonrelativistic free-free gaunt factor incorporating plasma screening. High Energy Density Physics, 10:61 - 69, 2014

[3] Carlos A. Iglesias. XUV absorption by solid-density aluminum. High Energy Density Physics, 6(3):311 - 317, 2010.

[4] B. Kettle, T. Dzelzainis, S. White, L. Li, B. Dromey, M. Zepf, C. L. S. Lewis, G. Williams, S. Künzel, M. Fa- jardo, H. Dacasa, Ph. Zeitoun, A. Rigby, G. Gregori, C. Spindloe, R. Heathcote, and D. Riley. Experimental measurements of the collisional absorption of XUV radiation in warm dense aluminium. Phys. Rev. E, 94:023203, Aug 2016.

[5] Sam M. Vinko, Gianluca Gregori, Michael P. Desjarlais, Bob Nagler, Thomas J. Whitcher, Richard W. Lee, Patrick Audebert, and Justin S. Wark. Free-free opacity in warm dense aluminum. High Energy Density Physics, 5(3):124 - 131, 2009.

[6] Balazs F. Rozsnyai. Solar opacities. Journal of Quantitative Spectroscopy and Radiative Transfer, 71(26):655663, 2001. Radiative Properties of Hot Dense Matter.

[7] S. Mazevet, L.A. Collins, N.H. Magee, J.D. Kress, 
and J.J. Keady. Quantum molecular dynamics calculations of radiative opacities. Astronomy \& Astrophysics, 405(1):L5-L9, 2003.

[8] G. Fontaine, P. Brassard, and P. Bergeron. The potential of white dwarf cosmochronology. Publications of the Astronomical Society of the Pacific, 113(782):409, 2001.

[9] Hendrik Anthony Kramers. Xciii. on the theory of x-ray absorption and of the continuous x-ray spectrum. The London, Edinburgh, and Dublin Philosophical Magazine and Journal of Science, 46(275):836-871, 1923.

[10] Robert R. Johnston. Free-free radiative transitionsa survey of theoretical results. Journal of Quantitative Spectroscopy and Radiative Transfer, 7(6):815 - 835, 1967.

[11] M. Nakagawa, Y. Kohyama, and N. Itoh. Relativistic free-free gaunt factor of the dense high-temperature stellar plasma. apjs, 63:661-684, March 1987.

[12] J. M. Green. The free-free gaunt factor in an ionized medium. Research memorandum of the RAND corporation, AD:214846, 1958.

[13] C. E. Starrett and D. Saumon. Electronic and ionic structures of warm and hot dense matter. Phys. Rev. E, 87:013104, Jan 2013.

[14] C.E. Starrett and D. Saumon. A simple method for determining the ionic structure of warm dense matter. High Energy Density Physics, 10:35 - 42, 2014.

[15] C.E. Starrett. Kubo-greenwood approach to conductivity in dense plasmas with average atom models. High Energy Density Physics, 19:58 - 64, 2016.

[16] Ryogo Kubo. Statistical-mechanical theory of irreversible processes. i. general theory and simple applications to magnetic and conduction problems. Journal of the Physical Society of Japan, 12(6):570-586, 1957.

[17] D.A. Greenwood. The boltzmann equation in the theory of electrical conduction in metals. Proceedings of the Physical Society, 71(4):585, 1958.

[18] W.R. Johnson, C. Guet, and G.F. Bertsch. Optical properties of plasmas based on an average-atom model. Journal of Quantitative Spectroscopy and Radiative Transfer, 99(13):327 - 340, 2006. Radiative Properties of Hot Dense Matter.

[19] Peter Hakel and David P. Kilcrease. CHEMEOS: A new chemical-picture-based model for plasma equationof-state calculations. In AIP Conference Proceedings, volume 730, pages 190-199. AIP, 2004.

[20] N. H. Magee, J. Abdallah, J. Colgan, P. Hakel, D. P. Kilcrease, S. Mazevet, M. Sherrill, C. J. Fontes, and H. L. Zhang. Los Alamos opacities: Transition from LEDCOP to ATOMIC. AIP Conference Proceedings, 730(1):168179, 2004

[21] P. Hakel, M.E. Sherrill, S. Mazevet, J. Abdallah Jr., J. Colgan, D.P. Kilcrease, N.H. Magee, C.J. Fontes, and H.L. Zhang. The new Los Alamos opacity code ATOMIC. Journal of Quantitative Spectroscopy and Radiative Transfer, 99(13):265 - 271, 2006. Radiative Properties of Hot Dense Matter.

[22] J. Colgan, D. P. Kilcrease, N. H. Magee, M. E. Sherrill, J. Abdallah Jr., P. Hakel, C. J. Fontes, J. A. Guzik, and K. A. Mussack. A new generation of Los Alamos opacity tables. The Astrophysical Journal, 817(2):116, 2016.

[23] Christopher John Fontes, H.L. Zhang, J. Abdallah Jr., Robert Edward Holmes Clark, David Parker Kilcrease, J. Colgan, Robert T. Cunningham, Peter Hakel, N.H. Magee, and Manolo Edgar Sherrill. The Los Alamos suite of relativistic atomic physics codes. Journal of Physics B:
Atomic, Molecular and Optical Physics, 48(14):144014, 2015.

[24] R. Piron and T. Blenski. Variational average-atom in quantum plasmas (VAAQP)-application to radiative properties. High Energy Density Physics, 9(4):702-710, 2013.

[25] François Perrot and M. W. C. Dharma-wardana. Electrical resistivity of hot dense plasmas. Phys. Rev. A, 36:238-246, Jul 1987.

[26] B.J.B. Crowley and J.W. Harris. Modelling of plasmas in an average-atom local density approximation: the CASSANDRA code. Journal of Quantitative Spectroscopy and Radiative Transfer, 71(26):257 - 272, 2001. Radiative Properties of Hot Dense Matter.

[27] F Perrot. Correlated fluctuations of electron populations in high temperature plasmas. Physica A: Statistical Mechanics and its Applications, 150(2):357-370, 1988.

[28] A.A. Ovechkin, P.A. Loboda, V.G. Novikov, A.S. Grushin, and A.D. Solomyannaya. Reseos a model of thermodynamic and optical properties of hot and warm dense matter. High Energy Density Physics, 13:20 - 33, 2014.

[29] C. E. Starrett, J. Daligault, and D. Saumon. Pseudoatom molecular dynamics. Phys. Rev. E, 91:013104, Jan 2015.

[30] A.A. Ovechkin, P.A. Loboda, and A.L. Falkov. Transport and dielectric properties of dense ionized matter from the average-atom reseos model. High Energy Density Physics, 20:38 - 54, 2016.

[31] W.R. Johnson. Low-frequency conductivity in the average-atom approximation. High Energy Density Physics, 5(12):61 - 67, 2009

[32] M. Yu. Kuchiev and W. R. Johnson. Low-frequency plasma conductivity in the average-atom approximation. Phys. Rev. E, 78:026401, Aug 2008.

[33] A. Weiss, W. Hillebrandt, H.-C. Thomas, and H. Ritter. Cox and Giuli's Principles of Stellar Structure. 2004.

[34] R. Keenan, C. L. S. Lewis, J. S. Wark, and E. Wolfrum. Measurements of the XUV transmission of aluminium with a soft x-ray laser. Journal of Physics B: Atomic, Molecular and Optical Physics, 35(20):L447, 2002.

[35] B.L. Henke, E.M. Gullikson, and J.C. Davis. X-ray interactions: Photoabsorption, scattering, transmission, and reflection at $\mathrm{e}=50-30,000 \mathrm{ev}, \mathrm{z}=1-92$. Atomic Data and Nuclear Data Tables, 54(2):181 - 342, 1993.

[36] Center for X-ray Optics. X-ray interactions with matter. http://henke.lbl.gov/optical_constants/.

[37] E. M. Gullikson, P. Denham, S. Mrowka, and J. H. Underwood. Absolute photoabsorption measurements of $\mathrm{mg}, \mathrm{al}$, and si in the soft-x-ray region below the $l_{2,3}$ edges. Phys. Rev. B, 49:16283-16288, Jun 1994.

[38] D. B. Tanner, A. J. Sievers, and R. A. Buhrman. Farinfrared absorption in small metallic particles. Phys. Rev. B, 11:1330-1341, Feb 1975.

[39] D.J. Burrill, D.V. Feinblum, M.R.J. Charest, and C.E. Starrett. Comparison of electron transport calculations in warm dense matter using the Ziman formula. High Energy Density Physics, 19:1 - 10, 2016.

[40] P.A. Sterne, S.B. Hansen, B.G. Wilson, and W.A. Isaacs. Equation of state, occupation probabilities and conductivities in the average atom Purgatorio code. High Energy Density Physics, 3(12):278 - 282, 2007. Radiative Properties of Hot Dense Matter. 